\title{
Bunch-by-bunch measurements of the betatron tune and the synchronous phase and their applications to beam dynamics at KEKB
}

\author{
T. Ieiri, K. Akai, H. Fukuma, T. Kawamoto, E. Kikutani, and M. Tobiyama \\ KEK, 1-1 Oho, Tsukuba, Ibaraki 305-0801, Japan \\ (Received 1 August 2002; published 27 September 2002)
}

\begin{abstract}
The betatron tune and the synchronous phase of individual bunches were measured at KEKB, an asymmetric electron/positron circular collider. The measurement was performed under three conditions: a single bunch, and multibunches with and without collision. The coupling impedance of the rings can be estimated from the current dependence of the parameters of a single bunch. The betatron tune along a positron-bunch train indicates variations of the electron cloud density that cause electron cloud instability. Comparing the betatron tune between colliding and noncolliding bunches, the beam-beam tune shift was investigated in regular operation. The coherent beam-beam parameter was evaluated in the two-ring collider with unequal tunes. Transient beam-loading effects were experimentally demonstrated by a phase shift along a bunch train in both rings. A rapid advance in the beam phase was observed, which is considered to be due to the effects of longitudinal wakes.
\end{abstract}

DOI: 10.1103/PhysRevSTAB.5.094402

PACS numbers: 29.27.Bd

\section{INTRODUCTION}

KEKB [1,2] is a multibunch, high-current, electron/ positron collider for $B$ meson physics. The collider consists of two storage rings: the Low Energy Ring (LER) for a $3.5 \mathrm{GeV}$ positron beam and the High Energy Ring (HER) for $8 \mathrm{GeV}$ electrons. Both rings store more than 1000 bunches, where the harmonic number is 5120 with an rf frequency of $509 \mathrm{MHz}$. Bunches are stored in two rings with a 4-bucket spacing (8 ns) in usual physics run, forming a single train followed by an empty gap that occupies $4.5 \%$ of the circumference. The two beams collide at one interaction point (IP) with a horizontal crossing angle of $22 \mathrm{mrad}$. The main machine parameters are listed in Table I.

The LER has suffered from a blowup in the vertical beam size since the early stage of commissioning [3]. The blowup is caused by electron clouds [4,5]. In order to confine the electrons to the vicinity of the chamber wall, permanent magnets with $\mathrm{C}$-shaped yokes were attached to the surface of the vacuum chamber. However, the permanent magnets were not sufficiently effective for curing the blowup. Solenoids were installed in place of the permanent magnets in September 2000, and they were effective to weaken the blowup. Therefore, we added solenoids in all spaces where installation was feasible [6]. Owing to the solenoids and precise adjustment of the machine parameters, more than $70 \%$ of the target luminosity of $1.0 \times 10^{34} \mathrm{~cm}^{-2} \mathrm{~s}^{-1}$ has been achieved by the summer shutdown in 2002.

Although all bunches stored have the same intensity, they do not all have equal orbits, equal sizes, nor the same betatron tunes. There are several effects that change such beam parameters bunch to bunch. First, a head bunch

TABLE I. Machine parameters used in the measurements.

\begin{tabular}{lccc}
\hline \hline \multicolumn{1}{c}{ Parameter } & LER & HER & \\
\hline Beam energy, $E$ & 3.5 & 8.0 & $\mathrm{GeV}$ \\
Momentum spread, $\delta_{\epsilon}$ & 7.3 & 6.7 & $\times 10^{-4}$ \\
Horizontal emittance, $\epsilon_{x}$ & 18 & 24 & $\mathrm{~nm}$ \\
Betatron tune, $\nu_{x} / \nu_{y}$ & $45.51 / 43.57^{\mathrm{a}}$ & $44.52 / 41.58^{\mathrm{a}}$ & \\
Beta's at IP, $\beta_{x}^{*} / \beta_{y}^{*}$ & $59 / 0.65$ & $63 / 0.7$ & $\mathrm{~cm}$ \\
Momentum compaction, $\alpha$ & 3.4 & 3.4 & $\times 10^{-4}$ \\
Bunch length, $\sigma_{l}$ & $6.5-8.0$ & $6.5-7.0$ & $\mathrm{~mm}$ \\
Synchrotron tune, $\nu_{s}$ & 0.022 & 0.020 & \\
Particles per bunch, $N_{b}$ & $3.7-6.2$ & $3.1-4.2$ & $\times 10^{10}$ \\
Bunch spacing, $S_{b}$ & & & $\mathrm{~m}$ \\
Circumference, $C$ & & & $\mathrm{~m}$ \\
rf frequency, $f_{\mathrm{rf}}$ & & & $\mathrm{MHz}$ \\
Harmonic number, $h$ & & 3016 & \\
\hline \hline
\end{tabular}

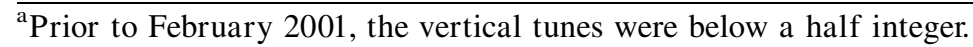


leaves electromagnetic fields behind it by the interaction with the environment and subsequent bunches are disturbed by the wake fields, depending on the bunch spacing. Next, the synchrotron light generated by the positron beam hits the wall of the chamber and makes photoelectrons. These electrons are attracted and accelerated by the subsequent positron bunches and form an electron cloud. The electron cloud density will gradually grow while proceeding along a train, becoming saturated when a balance is achieved between the production and the loss. On the other hand, the theory of the fast ion instability [7] predicts that ions created by the head bunch perturb the subsequent electron bunches in a train. Moreover, it is predicted that the gap causes a bunchby-bunch phase shift along a train due to the transient beam loading on the fundamental mode of rf cavities $[8,9]$. The phase shift causes the longitudinal displacement of the collision vertex, which may reduce the luminosity. Therefore, measurements of bunch-by-bunch parameters are strongly desired in order to understand the beam properties precisely. This investigation is important not only for KEKB but for all $e^{+} e^{-}$factories, present and future.

In order to measure the bunch-by-bunch beam parameters, a fast gate module that clearly selects a bunch in a bunch train has been developed and attached to the tune measurement system [2] and the four-dimensional beamposition monitor system [10]. The systems can measure the parameters of any bunch with a separation of more than three rf-buckets (6 ns). The betatron tune and the longitudinal position of individual bunches have been measured using the gated monitor systems in a single bunch and in multibunch operation. Comparing the beam parameters of collision bunches with those of noncollision bunches, the effects of the beam-beam interaction can be evaluated.

The next section describes the physical backgrounds related to the betatron tune and the synchronous phase. Section III briefly describes the systems for measuring the tune and the phase. Section IV presents the results measured both in a single bunch and in multibunches, including the colliding mode. Section $\mathrm{V}$ discusses two topics: the threshold for the electron cloud instability and the coherent beam-beam tune-shift parameter.

\section{PHYSICAL BACKGROUNDS}

Multibunch beam dynamics contains that of a single bunch. Thus, it is necessary to understand the beam properties in a single bunch, which are related to the broadband impedance, in order to investigate bunch-bybunch beam dynamics. This section deals with currentdependent tune shift of a single bunch, tune shift due to an electron cloud, beam-beam tune shift, and phase shift due to transient beam loading.

\section{A. Tune shift due to the impedance}

A bunch interacts with a vacuum chamber object and generates wake fields. These wake fields can act back on the bunch itself and affect the beam dynamics. A bunch oscillates with a tune $\nu=\nu_{\beta}+m \nu_{s}$, where $\nu_{\beta}$ is the betatron tune, $\nu_{s}$ is the synchrotron tune, and $m=$ $0, \pm 1, \pm 2, \ldots$. A shift of the current-dependent, coherent betatron tune of a bunch for the mode $m=0$ is given by [11]

$$
\frac{\Delta \nu}{I_{b}}=\frac{\langle\beta\rangle R\left\{Z_{\perp}\right\}}{4 \sqrt{\pi} \sigma_{l} E / e}
$$

where $I_{b}$ is the bunch current, $\langle\beta\rangle$ is the average value of the betatron function, $\sigma_{l}$ is the rms bunch length, $R$ is the average radius of the machine, and $\left\{Z_{\perp}\right\}$ is the effective coupling impedance. When the betatron tune shift is on the order of the synchrotron tune, a coupling between the modes $m=0$ and $m=-1$ causes an instability called the strong head-tail instability or the mode-coupling instability. When chromaticity is zero, the threshold current for the instability is given by

$$
I_{\mathrm{th}} \approx \frac{8}{\sqrt{\pi}} \frac{\nu_{s} \sigma_{l} E / e}{\langle\beta\rangle R\left\{Z_{\perp}\right\}} .
$$

The instability is a severe limitation on the single-bunch current in large storage rings with low beam energy and a low synchrotron tune, as in the case of the LER.

\section{B. Tune shift due to an electron cloud}

Since the effect of photoelectrons was first identified at the Photon Factory $[12,13]$, many efforts have been made to clarify the mechanism of the instability. Analytical work and simulations [14-16] have given an image of an electron cloud. Let us imagine a train of bunches passing through a curved region of a ring. Positron bunches emit the synchrotron light, which hits the vacuum chamber wall and produces photoelectrons. The photoelectrons are accelerated by subsequent bunches and yield secondary electrons. Since the polarity of the beam is positive, these electrons are gathered by the beam-produced field and form an electron cloud. The electron cloud interacts with positron bunches. A model described in Ref. [16] showed that the blowup of the vertical beam size observed in the LER was caused by strong head-tail instability. Using the two-particle model, the threshold of the electron density in a cloud for the blowup is given by

$$
\rho_{\text {th }}=\frac{2 \gamma \nu_{s}}{\pi r_{e} L\left\langle\beta_{y}\right\rangle},
$$

where $r_{e}$ is the classical electron radius, $\left\langle\beta_{y}\right\rangle$ is the average value of the vertical betatron function, $L$ is the length of the cloud, and $\gamma$ is the relativistic factor.

Experimentally, the density of the electrons in a cloud can be estimated from a shift of the betatron tune under 
the hypothesis that the electric fields due to the electron cloud result in a shifting of the coherent betatron tune of positron bunches. The tune shift due to the cloud is given by [17]

$$
\Delta \nu_{x(y)}=\frac{r_{e} \rho_{0}\langle\beta\rangle_{x(y)} L}{2 \gamma},
$$

under the condition that the majority of the electrons in the cloud are not disturbed during the passage of a positron bunch. Here, $\rho_{0}$ is the average density of the electron cloud and $\left\langle\beta_{x(y)}\right\rangle$ is the average value of the horizontal or vertical betatron function. Combining Eqs. (3) and (4), the threshold for the blowup in the tune shift is simply given by

$$
\Delta \nu_{\mathrm{th}} \approx \frac{\nu_{s}}{\pi} .
$$

This expression shows that the threshold is determined by the synchrotron tune alone. This condition is similar to that for the strong head-tail instability in a single bunch. A synchrotron tune of 0.022 results in a threshold of 0.007 in the tune shift.

\section{Beam-beam tune shift}

The performance of the beam collision can be evaluated by the beam-beam tune-shift parameter. When electron and positron beams collide, the space charge force of one of the beams gives the other beam a transverse kick and consequently changes the betatron tune. The beam-beam interaction introduces a new set of two betatron tunes by mixing two unperturbed tunes. We call these two new modes the higher tune $(H)$ and the lower tune $(L)$ modes; both modes are affected by the beambeam interaction, unlike the $\Sigma$ and $\pi$ modes in a singlering collider. Assuming the rigid Gaussian model, the resultant tunes in the horizontal or the vertical plane are expressed by [18]

$$
\begin{aligned}
\cos \mu_{q H} & +\cos \mu_{q L}-\left(\cos \mu_{q 0}^{+}+\cos \mu_{q 0}^{-}\right) \\
& =2 \pi\left(\Xi_{q}^{+} \sin \mu_{q 0}^{+}+\Xi_{q}^{-} \sin \mu_{q 0}^{-}\right),
\end{aligned}
$$

with

$$
\Xi_{q}^{ \pm}=\frac{N_{b}^{\mp} r_{e}}{\gamma^{ \pm}} \frac{\beta_{q}^{* \pm}}{2 \pi \Sigma_{q}\left(\Sigma_{x}+\Sigma_{y}\right)} R_{q}^{ \pm} .
$$

Here, $\mu_{q 0}^{ \pm}$is the betatron phase advance without collision and $R_{q}^{ \pm}$is the reduction factor for the beam-beam parameter due to the crossing angle and the hourglass effect, where the subscript 0 means noncollision, the other subscript $q$ stands for either the $x$ or $y$ plane, and the superscript \pm denotes positron and electron bunches. The unperturbed tune is given by $\nu_{0}^{ \pm}=\mu_{0}^{ \pm} / 2 \pi$. The coherent beam-beam parameter $\Xi_{q}^{ \pm}$is a function of the effective beam size $\Sigma_{q}=\sqrt{\left(\sigma_{q}^{+}\right)^{2}+\left(\sigma_{q}^{-}\right)^{2}}$, and it is half of the incoherent beam-beam parameter $\xi_{q}$, when both sizes are equal; that is, $\xi_{q} \approx \Xi_{q}^{+}+\Xi_{q}^{-}$. The perturbed tunes are calculated as a function of the coherent beam-beam parameter in the case of $\Xi_{q}^{+}=\Xi_{q}^{-}$, as shown in Fig. 1. Both tunes are shifted to higher values as the beam-beam parameter increases. The tune of the $H$ mode linearly increases, while the $L$ mode tune tends to saturate. The total beam-beam tune shift defined by $\Delta \nu_{q b-b}=\nu_{q H}+$ $\nu_{q L}-\nu_{q 0}^{+}-\nu_{q 0}^{-}$can be obtained as a function of the sum of the coherent beam-beam parameters $\Xi_{q}^{+}+\Xi_{q}^{-}$, as shown in Fig. 2. The relation is not linear and depends on the unperturbed tunes. It is noted that the Yokoya factor [19] is not included in Eq. (6). The tune of each mode was also calculated in the case of $\Xi_{q}^{+} \neq \Xi_{q}^{-}$under the condition that the value of $\Xi_{q}^{+}+\Xi_{q}^{-}$was kept constant. When $\Xi_{q}^{+} \succ \Xi_{q}^{-}$, the tune of the $H$ mode decreased, whereas that of the $L$ mode increased, compared with the case of $\Xi_{q}^{+}=\Xi_{q}^{-}$. On the other hand, in the case of $\Xi_{q}^{+} \prec$ $\Xi_{q}^{-}$, the tune of the $H$ mode increased and that of the $L$ mode decreased, compared to the case of $\Xi_{q}^{+}=\Xi_{q}^{-}$. Even if the ratio $\Xi_{q}^{+} / \Xi_{q}^{-}$changed from 0.2 to 5.0, the total beam-beam tune shift settled within less than $\pm 7 \%$. This means that the sum of the coherent beam-beam parameters $\Xi_{q}^{+}+\Xi_{q}^{-}$is approximately determined by the total beam-beam tune shift $\Delta \nu_{q b-b}$. After determination of $\Xi_{q}^{+}+\Xi_{q}^{-}$, each beam-beam parameter can be estimated from the shift of the individual tunes.

Important parameters are derived from the beambeam parameters. The luminosity without an offset error is given by

$$
L=\frac{N_{b}^{+} N_{b}^{-} f_{0}}{2 \pi \Sigma_{x} \Sigma_{y}} R_{L}
$$

where $f_{0}$ is the revolution frequency and $R_{L}$ is the reduction factor of the luminosity due to the crossing angle and the hourglass effect. Assuming flat beams with equal betas, that is, $\beta_{x, y}^{+}=\beta_{x, y}^{-}=\bar{\beta}_{x, y}^{*}$ and $\beta_{x} \gg \beta_{y}$, the luminosity is proportional to the vertical beam-beam parameter and represented by

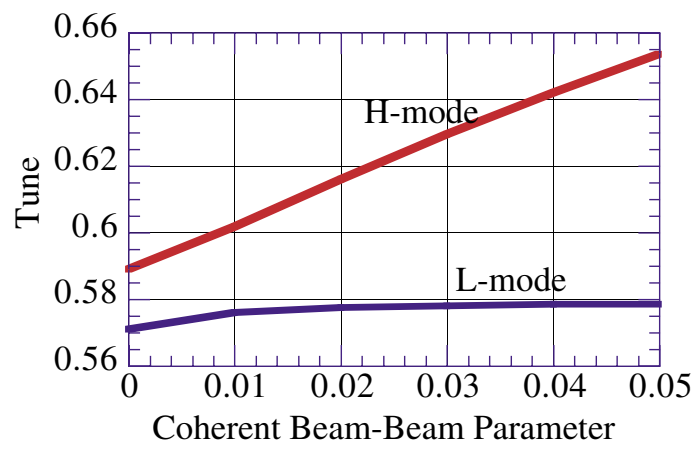

FIG. 1. (Color) Tunes as a function of the coherent beam-beam parameter, assuming $\Xi_{q}^{+}=\Xi_{q}^{-}$, where the unperturbed tunes of $\nu_{q 0}^{+}=0.571$ and $\nu_{q 0}^{-}=0.589$ are used. 


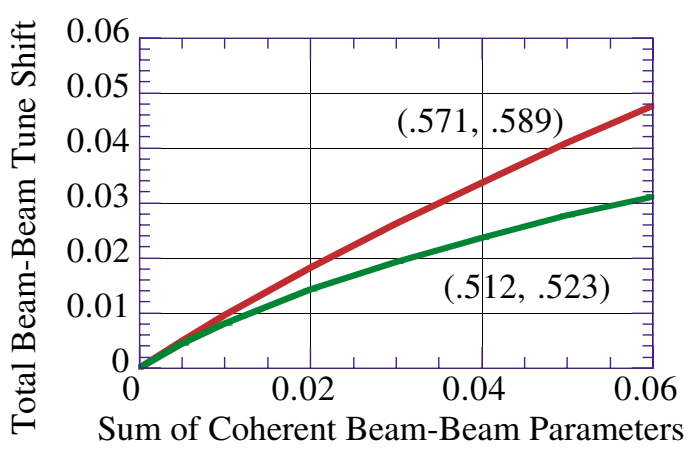

FIG. 2. (Color) Relation between the total beam-beam tune shift $\Delta \nu_{q b-b}$ and the sum of the coherent beam-beam parameters $\Xi_{q}^{+}+\Xi_{q}^{-}$, for two pairs of unperturbed tunes. The Yokoya factor is not included.

$$
L=\frac{f_{0} N_{b}^{+} \gamma^{+}}{r_{e}} \frac{T}{1+T} \frac{\Xi_{y}^{+}+\Xi_{y}^{-}}{\bar{\beta}_{y}^{*}} \frac{R_{L}}{\bar{R}_{y}^{*}} .
$$

Here, $\bar{R}_{y}^{*}=\left(R_{y}^{+}+R_{y}^{-}\right) / 2$ is the average reduction factor of the vertical beam-beam parameter and the parameter $T$ represented by the energy transparency factor $T=N_{b}^{-} \gamma^{-} / N_{b}^{+} \gamma^{+}$is larger than 1.0 at KEKB. The horizontal emittance is estimated from the horizontal beambeam parameter. From Eq. (7), we have

$$
\epsilon_{x}^{+}+\epsilon_{x}^{-}=\frac{r_{e}}{2 \pi \gamma^{-}} \frac{N_{b}^{+}(1+T)}{\Xi_{x}^{+}+\Xi_{x}^{-}} \bar{R}_{x}^{*}
$$

where $\beta_{x}^{+}=\beta_{x}^{-}$is assumed and the average reduction factor of the horizontal beam-beam parameter $\bar{R}_{x}^{*}=$ $\left(R_{x}^{+}+R_{x}^{-}\right) / 2$ is used.

\section{Phase shift of a bunch}

Accelerating cavities in a storage ring give energy to a circulating bunch to compensate for the radiation loss and parasitic loss. The synchronous phase of a bunch is determined by the balance between the cavity voltage and the total energy loss. The parasitic loss is caused by the impedance of the environment, represented by the loss factor $k(\sigma)$ depending on the bunch length. The shift in the synchronous phase due to the parasitic loss is given by

$$
V_{c} \sin \left(\phi_{s 0}+\Delta \phi_{s}\right)=\frac{U_{0}}{e}+k(\sigma) T_{0} I_{b}
$$

where $V_{c}$ is the cavity voltage, $\phi_{s 0}$ is the synchronous phase of a zero-current limit, $U_{0}$ is the radiation loss per turn, and $T_{0}$ is the revolution period. When the phase shift $\Delta \phi_{s}$ is small enough compared to unity, it is given by

$$
\frac{\Delta \phi_{s}}{I_{b}} \approx k(\sigma) \frac{T_{0}}{V_{c} \cos \phi_{s 0}} .
$$

The beam-phase advances as the bunch-current increases and the slope is proportional to the loss factor.
In a high-current and multibunch beam, the amplitude and phase of the accelerating voltage is modulated by the existence of a gap, since the beam loading is different between the gap part and the train part occupied by bunches. As a result, the synchronous position is shifted bunch to bunch along a train. Assuming that the cavity is operated at the optimum tuning, that the filling time of the cavity $T_{f}$ is much longer than the revolution period $T_{0}$, i.e., $T_{f} / T_{0} \gg 1$, and that the synchronous phase $\phi_{s} \approx$ 0 , the amount of the beam-phase difference between the head and tail bunches is approximately given by [8]

$$
\Delta \phi_{\mathrm{ht}} \approx \frac{I_{t}}{2 V_{c}} \frac{R_{s}}{Q} \omega_{\mathrm{rf}} \Delta t
$$

where $I_{t}$ is the total beam current, $R_{s}$ is the shunt impedance, $Q$ is the $Q$ value of the cavity, $\omega_{\text {rf }}$ is the angular $\mathrm{rf}$ frequency, and $\Delta t$ is the length of the gap. In the case of $\mathrm{KEKB}$, however, this simple formula cannot be precisely applied; $T_{0} / \mathrm{T}_{f}=0.54$ for the normal-conducting cavities in the LER, and two different types of cavities (normal conducting and superconducting) are operated in the HER. Thus, the phase shift of each bunch was estimated by a simulation. The code used in the simulation was developed to study the performance of the beam-cavity system of KEKB including feedback loops [9]. When the phase along a train is different between the two rings, it gives rise to a longitudinal displacement of the collision vertex along a train, as given by

$$
\Delta L_{\mathrm{vertex}}=\frac{c}{\omega_{\mathrm{rf}}} \frac{\left(\phi_{\mathrm{HER}}-\Phi_{\mathrm{LER}}\right)}{2},
$$

where $c$ is the speed of light.

\section{MEASUREMENT SYSTEMS}

There are two methods to measure the bunch-by-bunch parameters. The first method is to sample all bunches continuously, where the signal processing finishes before the next bunch comes. This continuous sampling method is used in the bunch-by-bunch feedback system [20] and has the merit that all bunches in a ring are processed simultaneously. However, it is difficult to obtain a bunchcurrent independent signal. The other method is to select a specific bunch using a high-speed gate. Compared with the continuous sampling method, this method allows the execution of signal processing over a revolution period. Thus, a precise measurement is expected for the bunch parameters.

\section{A. Gate module}

A gating circuit for selecting a bunch is required to have following specifications: fast switching, high isolation, and a wide dynamic range. Commercially available GaAs switches are used for the gate. Two switches are connected in a series to improve the isolation, and 


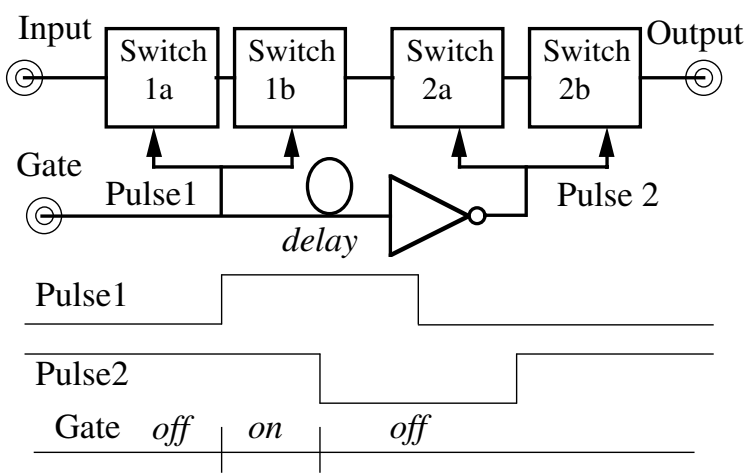

FIG. 3. Schematic of the gate module.

they are connected in a series again, as shown in Fig. 3. Two pulses for switching are prepared for each pair of switches to avoid a ringing problem at the transition of on-to-off states, which are complementary to each other, but one of the signals is delayed by a cable. The length of the cable determines the gate duration. A gate duration of $6 \mathrm{~ns}$ was obtained with an insertion loss of $3 \mathrm{~dB}$. The isolation on a bench was confirmed to be about $60 \mathrm{~dB}$ at $1 \mathrm{GHz}$. However, the isolation measured with a beam signal was $32 \mathrm{~dB}$ in the HER and $35 \mathrm{~dB}$ in the LER. Some noises would therefore be mixed in the beam signal picked up by button electrodes and transmitted through the cables. The intensity response of the gate showed good linearity within 5\% with the bunch current over a range of 0.2 to $1.4 \mathrm{~mA}$. The dynamic phase response, however, showed a large deviation from the expected value as the current increased, and the direction of the deviation depended on the polarity of the input pulse. Monitoring the bunch intensity should compensate for the phase deviations. Although some noises were observed at switching, they were canceled out by storing the data in a memory and subtracting them. The performance of the gate module is summarized in Table II.

\section{B. Gated tune measurement system}

The gate module was applied to a tune measurement system. We call this system equipped with the gate module "the gated tune measurement system" [2]. A block diagram shown in Fig. 4 schematically explains the system. The frequency of the output signal of the tracking generator is swept over the range corresponding to a fractional part of the betatron tune $(1 \sim 50 \mathrm{kHz})$ and

TABLE II. Performance of the gate module.

\begin{tabular}{lc}
\hline \hline Frequency range & dc to $6 \mathrm{GHz}$ \\
On/off isolation & $60 \mathrm{~dB}$ at $1 \mathrm{GHz}$ on bench \\
& 32 to $35 \mathrm{~dB}$ with beam \\
Rise time & $3 \mathrm{~ns}$ \\
Dynamic range & $+29 \mathrm{dBm}$ at $1 \mathrm{~dB}$ compression \\
\hline \hline
\end{tabular}

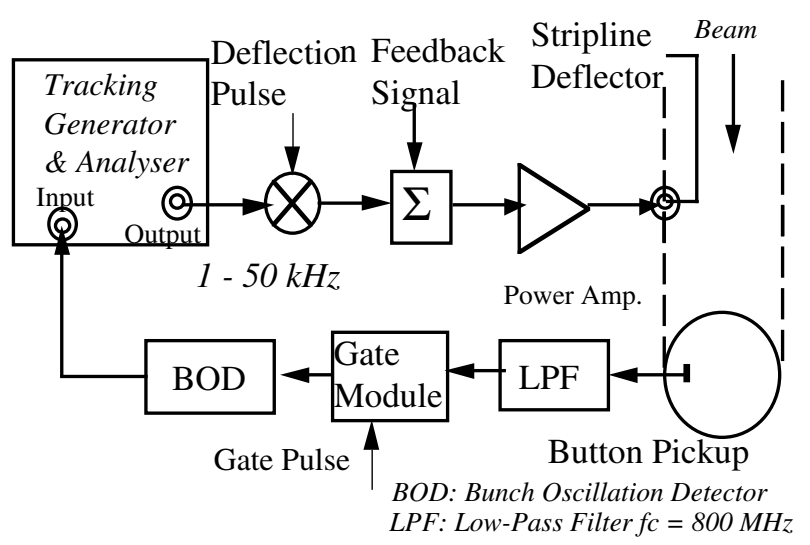

FIG. 4. Schematic of the gated tune measurement system.

the amplitude is modulated by a pulse synchronized with a revolution signal of $99.39 \mathrm{kHz}$. The amplitudemodulated pulse having a width of about $50 \mathrm{~ns}$ is combined with a feedback signal, and the combined signals are guided to deflector electrodes after amplification. The deflector consists of four strip-line electrodes mounted in a vacuum chamber. The timing of the deflection pulse is adjusted using a delay module with an interval of $2 \mathrm{~ns}$, one cycle of the rf signal.

A bunch signal picked up by a button electrode is selected by the gate module. The bunch oscillation detector (BOD) shown in Fig. 4 extracts intensity-independent oscillatory components from a gated signal, owing to an automatic gain control loop which is effective for variations of a beam intensity and an orbit, but not effective for bunch oscillations. The detected signal is put into the tracking analyzer.

The noise level displayed on the tracking analyzer corresponds to an oscillation amplitude of less than $10 \mu \mathrm{m}$, which is less than the rms vertical beam size at the pickup electrode. The resolution of the tune measurement is mainly determined by the bandwidth of the tracking analyzer and it is estimated to be \pm 0.0004 in the present configuration. A smoothing technique for the spectrum may improve the resolution.

As mentioned before, the system is coupled with the transverse feedback system, where the loop is always closed. The transverse feedback enhances the damping of the betatron oscillation to cope with instabilities, which results in lowering the $Q$ value in the tune spectrum. The feedback is not compatible with a precise tune measurement. Thus, a gate inserted on the feedback line cuts off the feedback power to a single bunch for tune measurement using a fast switch. This gating enables us to measure the beam-beam tune-shift parameter described in Sec. IV D.

\section{Gated phase measurement system}

Another application of the gate module is the fourdimensional beam-position monitor [10], which employs 


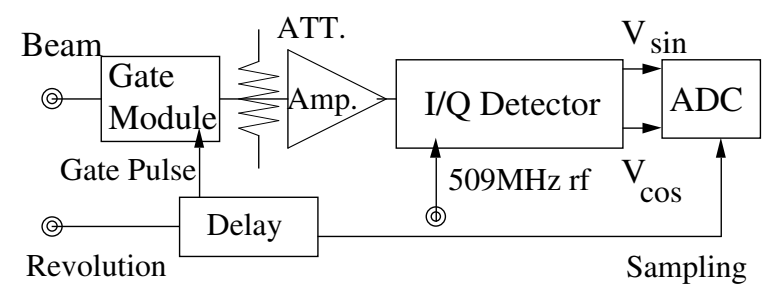

FIG. 5. Schematic of bunch phase detection.

an I/Q (in-phase and quadrature phase) demodulator working at an rf frequency of $509 \mathrm{MHz}$. A beam signal picked up by a button electrode is gated by a pulse synchronized with the revolution signal. The two detected signals, represented by $V_{\text {sin }}$ and $V_{\cos }$ as shown in Fig. 5, are sampled by the revolution pulse and stored in the memory of an ADC (analog-digital converter) module. The ratio of amplitudes of two orthogonal components gives a phase difference between the beam and the reference rf signal. The phase difference is given by

$$
\phi_{b}-\phi_{\mathrm{rf}}=\tan ^{-1}\left(-\frac{V_{\mathrm{sin}}}{V_{\cos }}\right)
$$

where $\phi_{b}$ and $\phi_{\mathrm{rf}}$ are the beam and the reference $\mathrm{rf}$ phases, respectively. Assuming that the rf phase is constant, the beam phase is obtained from the amplitude ratio. The amplitude data are sampled with a 14-bit ADC every turn $(10 \mu \mathrm{s})$ and are averaged over 32000 turns. We have confirmed that the average values in the phase difference were settled within $\pm 0.1^{\circ}$ under a constant intensity. The phase resolution corresponds to a time resolution of less than $1 \mathrm{ps}$. A drift of the phase, however, was observed over a long time.

\section{MEASUREMENTS}

\section{A. Tune shift in a single bunch}

The betatron tune of a single bunch was measured in the LER. The chromaticity was set to a low value in order to reduce the chromatic effects. The measured horizontal and vertical chromaticities were 2.2 and 0.8 , respectively. The measured fractional parts of the betatron tune for the mode $m=0$ are plotted as a function of the bunch current in Fig. 6. Although the tune shift depends on the bunch length, the tune decreases almost linearly with the current. The bunch length actually varied from 5 to $7 \mathrm{~mm}$ [21]. A tune shift of $\Delta \nu_{y} / I_{b}=-0.0034 / \mathrm{mA}$ to $-0.0042 / \mathrm{mA}$ was obtained in the vertical plane. The corresponding effective impedance is 80.6 to $99.6 \mathrm{k} \Omega / \mathrm{m}$ at $\sigma_{l}=5.0 \mathrm{~mm}$ and is 112.8 to $139.4 \mathrm{k} \Omega / \mathrm{m}$ at $\sigma_{l}=7.0 \mathrm{~mm}$. The horizontal tune shift was $\delta \nu_{x}=$ $-0.0010 / \mathrm{mA}$ to $-0.0015 / \mathrm{mA}$. These values for both planes are significantly larger than the design value of $-0.0004 / \mathrm{mA}[1]$.
Since a circular beam pipe is used in the LER, the transverse impedances of both planes would be the same if the components in the vacuum chamber were symmetrical. A candidate for breaking the symmetry is movable collimators [22]. In order to investigate the effect of the collimators, measurements were also performed with the collimators fully opened in both planes. The vertical tune shift was reduced to $-0.0014 / \mathrm{mA}$, as shown in Fig. 6(b). Therefore, more than half of the vertical impedance in the LER must be due to these collimators, when they are at their usual positions. The horizontal tune shift, however, did not change significantly. The horizontal collimators were normally opened wider than the vertical ones.

\section{B. Bunch-by-bunch tune of multibunch positron beam}

The betatron tune was measured along a bunch train without collision in the LER without C-yoke permanent magnets nor solenoids, where a train contained 40 bunches placed with a 4-bucket spacing ( $8 \mathrm{~ns})$. We observed three peaks in the vertical tune spectrum of each bunch on the tracking scope. The central peak corresponded to a pure betatron frequency represented by the $m=0$ dipole mode. The two sideband peaks indicated synchrobetatron modes represented by the modes $m=1$ and $m=-1$. Comparing the spectrum of a tail bunch with that of a head bunch, we observed that the peaks shifted towards a higher tune with widening of the spectrum. Figure 7 shows a fractional part of the vertical tunes as a function of the bucket number divided by 4 along a train. All of the tunes rapidly increase at the leading part and tend to saturate after the 10th bunch, or $72 \mathrm{~ns}$ behind the head bunch. The behavior suggests that the force causing the bunch-by-bunch tune shift mainly comes from outside of the positron bunches. It is noted that the peaks never couple with each other, unlike the transverse modecoupling instability observed in a single bunch.

The nature of the bunch-by-bunch tune shift was investigated in more detail. We experimentally found [4] that the rate of increase of the tune at the leading part in a train is primarily determined by the line density of bunches, that is, the bunch current divided by the spacing under a fixed chromaticity. Comparing two measurements with the transverse bunch feedback [20] on and off, it was confirmed that the feedback made only a common shift in the tune for all bunches. When the excitation amplitude was increased over a range of more than $20 \mathrm{~dB}$, the tune settled to a value within the measurement error. Thus, the nonlinear effect of the deflection amplitude is small. Figure 8 shows the difference in the vertical tune between the head bunch and the tail bunch as a function of the vertical chromaticity. The tune difference linearly decreases with the chromaticity at a slope of $-6.6 \times$ $10^{-4}$. In a single bunch, however, such variations in the vertical tune depending on the chromaticity were not 
(a)

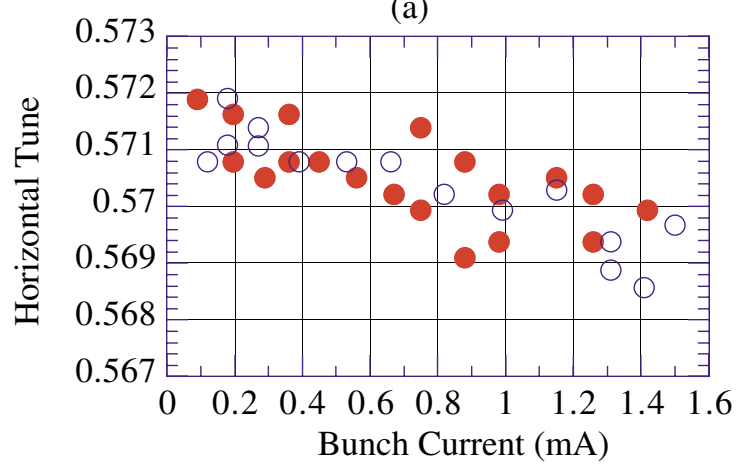

(b)

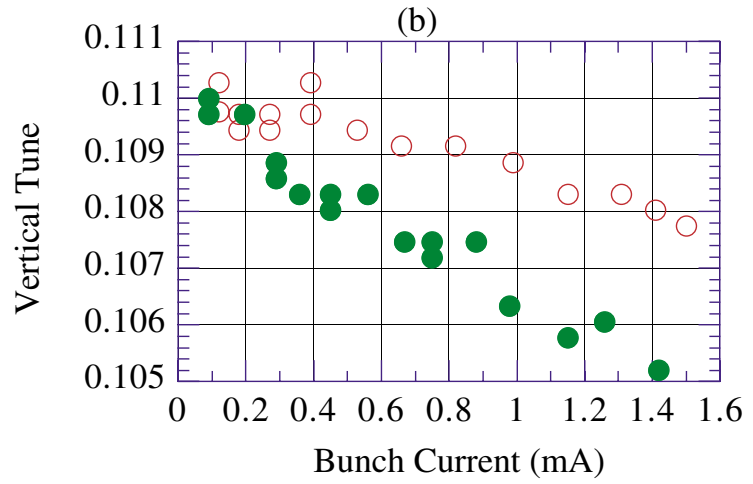

FIG. 6. (Color) Transverse tune versus bunch current in the horizontal plane (a) and the vertical plane (b). The results are shown with the collimators at their usual positions (shaded dots), and with the collimators fully opened (circles). Note that the vertical tune was below a half integer in this measurement.

observed. Thus, the variation in the tune observed in a bunch train is caused by the multibunch effect. On the other hand, it was experimentally found that the vertical size was suppressed by increasing the chromaticity [4]. These experimental results suggest that the chromaticity is a factor influencing the electron cloud instability.

Based on the understanding that solenoids are effective in controlling the electron cloud density, we compared the bunch-by-bunch tune with the solenoids on and off. In the measurements, the solenoid field of about $45 \mathrm{G}$ covered a region of $1200 \mathrm{~m}$, occupying half of the total arc sections of the LER. The train contained 32 bunches with a 4bucket spacing. We chose the tune of the head bunch as a reference. Since there was sufficient empty space of more than 2000 buckets in front of the bunch train, the tune of the head bunch should not be affected by the electron cloud. We did not need to take the effect of a tune shift due to transverse impedance into account, because it should equally act on all bunches. The results of the measurement are shown in Fig. 9. The tune shift grows rapidly at the leading part of the train and tends to saturate. According

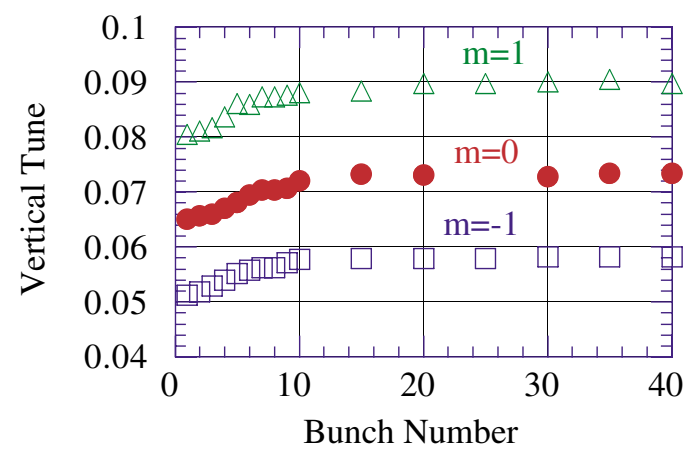

FIG. 7. (Color) Vertical tunes of the three modes $(m=0, m=$ 1 , and $m=-1$ ) along a train with a 4-bucket spacing in a fieldfree condition, measured in July 2000. The bunch current was $0.21 \mathrm{~mA}$, the vertical chromaticity was 11.2 , and the synchrotron tune was $\nu_{s}=0.0156$. to Eq. (4), the cloud density also grows rapidly at the leading part of the train. This phenomenon is common to the horizontal and vertical planes. When the solenoids are not active, equivalent to the field-free condition, the tune shift at the tail part is 0.004 in the horizontal plane and 0.0055 in the vertical plane at a bunch current of $0.3 \mathrm{~mA}$. These tune shifts correspond to a cloud density of $\rho_{0}=$ $5.9 \times 10^{11}\left(\mathrm{~m}^{-3}\right)$ in the horizontal plane and $8.1 \times$ $10^{11}\left(\mathrm{~m}^{-3}\right)$ in the vertical plane, where Eq. (4) is used with $L=3000 \mathrm{~m}$ and $\langle\beta\rangle_{x(y)}=11 \mathrm{~m}$. On the other hand, according to the simulation [15], the cloud density grows rapidly in the leading part of a train, from the head to $100 \mathrm{~ns}$ (50 buckets), and saturates at the tail part. The cloud density at the saturated region is about $12.0 \times$ $10^{11}\left(\mathrm{~m}^{-3}\right)$ at a bunch current of $0.5 \mathrm{~mA}$ in the fieldfree condition. The measurements and the simulation are consistent with each other, considering that the cloud density is approximately proportional to the bunch current.

The effects of the solenoids shown in Fig. 9 reveal that the horizontal tune shift was reduced to half, whereas the vertical tune was reduced by only $20 \%$ at the tail of the train. Though the variation in the vertical tune was small,

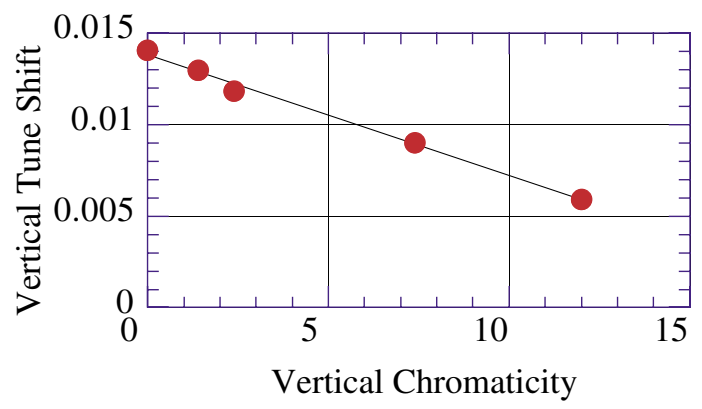

FIG. 8. (Color) Tune difference between a head bunch and a tail bunch in a train as a function of chromaticity; measurement was carried out with attachment of the $\mathrm{C}$-yoke magnets and at a bunch current of $0.8 \mathrm{~mA}$. 

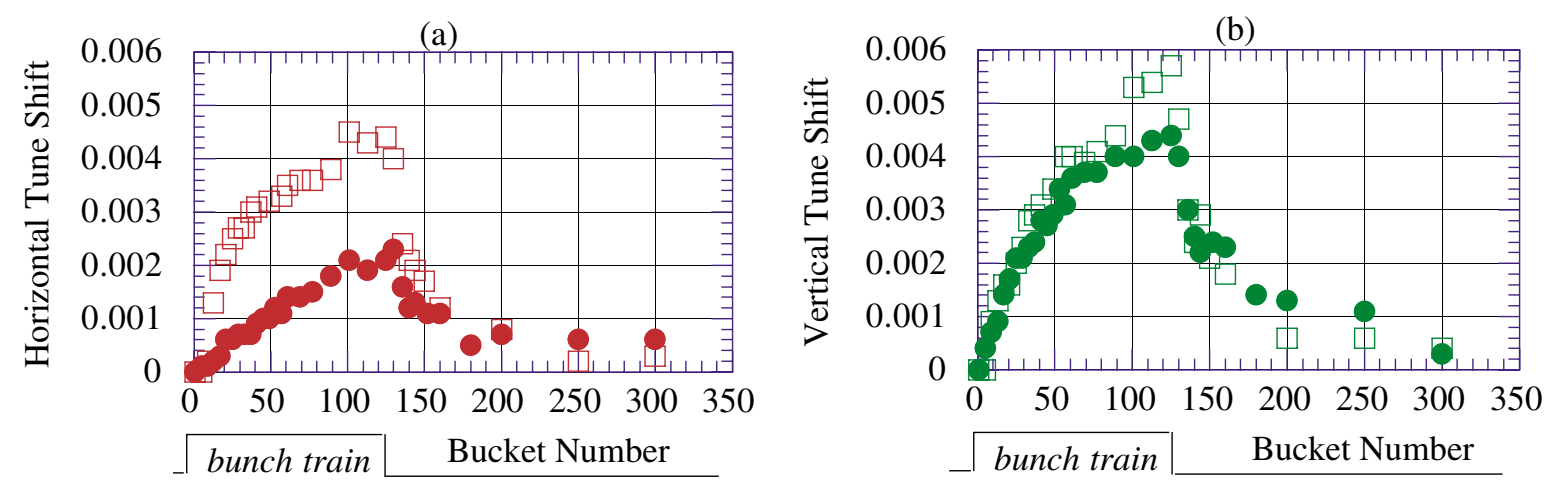

FIG. 9. (Color) Effects of solenoids on the bunch-by-bunch tune shifts, measured in April 2001; (a) is the horizontal tune shifts and (b) is the vertical tune shifts. The squares are the tune shifts with the solenoids off and the shaded dots are those with the solenoids on. The train contains 32 bunches with a 4-bucket spacing.

the shape of the spectrum changed and its width narrowed when the solenoid fields were applied. The reason for the asymmetric contribution of the solenoids is not clear. One candidate for the cause is the effect of the electrons in the lattice magnets, where the solenoids cannot be wound. Another simulation [23] indicates that multipacting of electrons occurs vertically in dipole magnets.

In order to investigate the decay of the electron cloud, a witness bunch was placed after the train and its position was changed. The decay of the tune shift is also shown in Fig. 9. Two steps of the decay can be seen in both directions: a fast decay with a time constant of less than $100 \mathrm{~ns}$ (50 buckets) and a slow decay with a long tail. The tails seem to be slightly enhanced when the solenoids are on.

\section{Tune of multibunch electron beam}

The tune of electron bunches along a train was measured without collision in the HER. The train contained 1223 bunches with a 4-bucket spacing. Figure 10 shows the tunes as a function of the bucket number for several beam currents, revealing that the measured tunes are almost constant along the train in both planes unlike the positron bunches. These results indicate that the tune shift due to the fast ion mechanism is negligible. However, the tune depends on the total beam current, where the dependence has different signs between the horizontal and vertical planes. The slopes are 0.026/A in the horizontal plane and $-0.034 / \mathrm{A}$ in the vertical plane. Since the measured data contain the single-bunch effect that causes a negative-slope dependence on the bunch current in both planes, we subtracted this effect to derive the multibunch effect. As a result, the slope of the horizontal tune shift was $0.030 / \mathrm{A}$ and that of the vertical tune shift was $-0.030 / \mathrm{A}$. This tune shift is caused by a quadrupole field generated by the resistivewall chambers having asymmetric cross sections [24]. Similar results were reported from the ESRF [25] and PEP-II [26]. This large tune shift requires careful management to maintain a constant tune during injection.

\section{Beam-beam tune shift}

Both rings are usually operated with a single bunch train followed by an empty gap. Bunches are spaced by $8 \mathrm{~ns}$, or four rf-buckets in the train. Additional bunches called pilot bunches are placed after the train, at different locations in each ring so that they do not collide with each other as illustrated in Fig. 11.
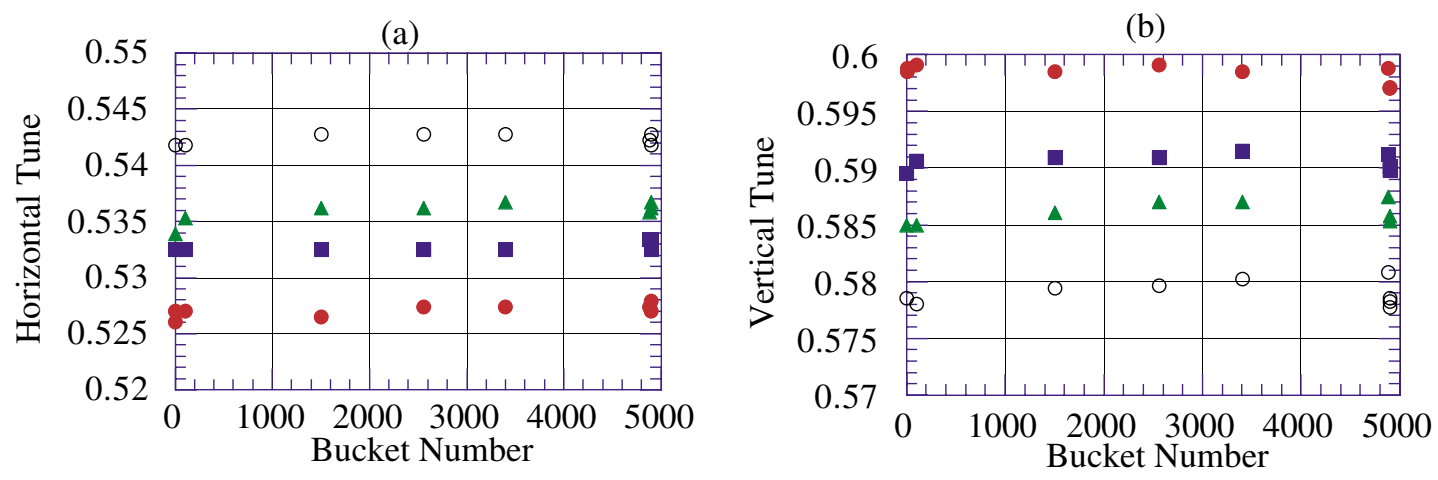

FIG. 10. (Color) Fractional part of the tunes along a train, with beam currents of $300 \mathrm{~mA}$ (shaded dots), $490 \mathrm{~mA}$ (squares), $620 \mathrm{~mA}$ (triangles), and $860 \mathrm{~mA}$ (circles). (a) is the horizontal tune and (b) is the vertical tune. 


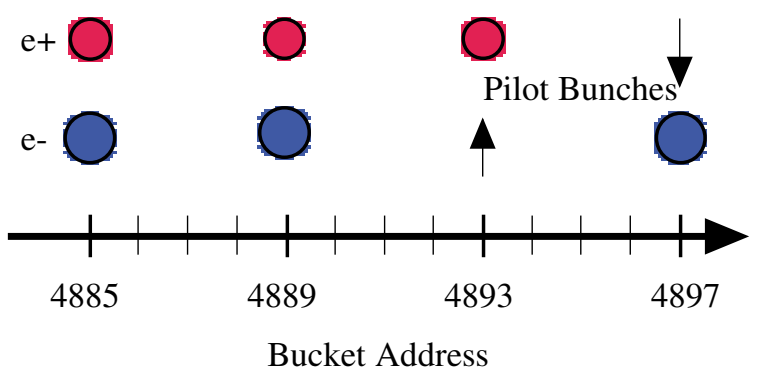

FIG. 11. (Color) Configuration of bunches at the tail of a train.

Tune measurement for the pilot bunches was performed during regular operation. It was experimentally verified that the bunch-by-bunch tune was equal at the tail part of the train, when bunches with equal intensity were equally spaced. Thus, the effects of the collision can be evaluated even when measuring different bunches. Typical spectra of colliding bunches are shown in Fig. 12. Two peaks in each horizontal spectrum are observed with a separation of 0.024. The horizontal tune with a large amplitude observed in the LER is equal to that in the HER, which corresponds to the horizontal $L$ mode. Both horizontal tunes are in the middle of those of the two pilot bunches separated by 0.01 . The second peak with a small amplitude corresponds to the horizontal $H$ mode. On the other hand, the vertical spectrum is broader than the horizontal one, especially in the HER. Although only one peak is seen in each vertical spectrum with a different tune, a

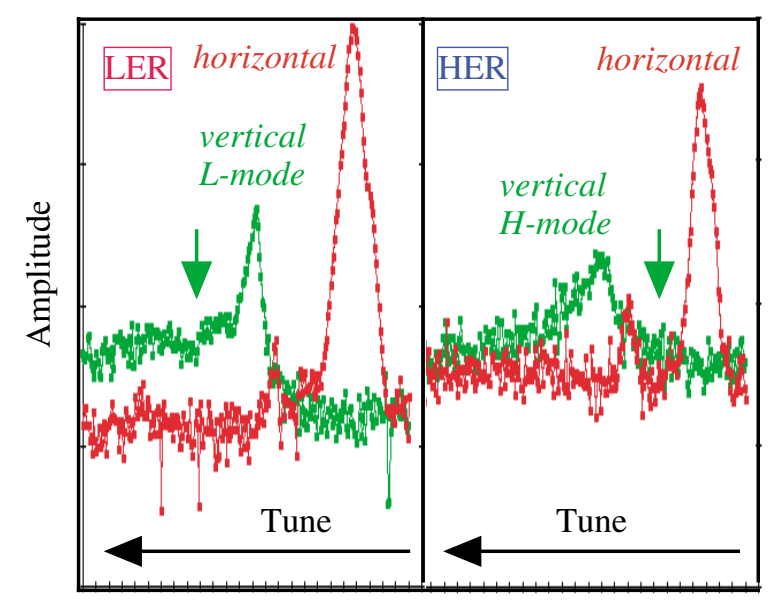

FIG. 12. (Color) Typical tune spectra of colliding bunches measured at bunch currents of $0.85 \mathrm{~mA}$ in the LER (left) and $0.63 \mathrm{~mA}$ in the HER (right). The vertical axis is amplitude with a full scale of $60 \mathrm{~dB}$ and the horizontal axis is frequency or tune with a full scale of 0.09 in the LER and 0.075 in the HER. The horizontal spectrum is shown in red and the vertical spectrum is shown in green. The green arrows indicate a tune of the other mode in the vertical spectrum. Note that the tune increases from right to left and that fractional tunes of the pilot bunches are $\nu_{x 0}^{+}=0.5124, \nu_{y 0}^{+}=0.5712, \nu_{x 0}^{-}=0.5221$, and $\nu_{y 0}^{-}=0.5903$.

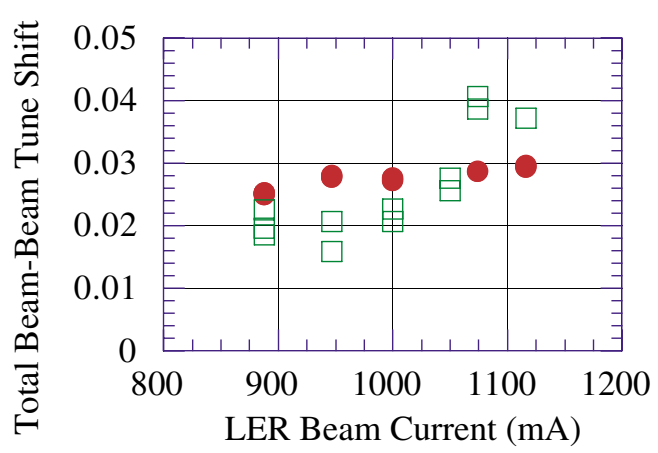

FIG. 13. (Color) Total beam-beam tune shifts as a function of the LER beam current. The shaded dots are horizontal tune shifts and the squares are vertical tune shifts. The data were obtained on March 25, 2002.

small discontinuity, indicated with a green arrow in Fig. 12, corresponding to a tune of the other mode can be seen in each spectrum.

The total beam-beam tune shift as a function of the LER beam current is shown in Fig. 13, when the tunes of the pilot bunches were $\nu_{x 0}^{+}=0.513, \nu_{y 0}^{+}=0.565, \nu_{x 0}^{-}=$ 0.512 , and $\nu_{y 0}^{-}=0.583$. The total tune shifts tend to increase with the beam current. The sum of the coherent beam-beam parameters is obtained from the total tuneshift data using the curves shown in Fig. 2. The horizontal beam-beam parameter $\Xi_{x}^{+}+\Xi_{x}^{-}$is estimated to be 0.04 to 0.05 , considering the Yokoya factor [19] of 1.31, since the horizontal tunes of the pilot bunches are nearly equal to each other in this case. On the other hand, the vertical beam-beam parameter is $\Xi_{y}^{+}+\Xi_{y}^{-}=0.018$ to 0.041 . Since the vertical tunes are separated by 0.02 , the Yokoya factor would be close to 1.0 [27]. The measured tune shifts are smaller in the $H$ mode and larger in the $L$ mode than those calculated using $\Xi_{q}^{+}=\Xi_{q}^{-}$, which suggests $\Xi_{y}^{+} \succ \Xi_{y}^{-}$in the vertical plane.

\section{E. Bunch-by-bunch phase in multibunches}

The beam phase was measured along a train during regular collision using the gate module. The train followed by a gap contained 1223 bunches with a 4-bucket spacing. The intensity of each bunch was uniform within $\pm 5 \%$. Since the intensity-dependent phase error of the gate module was estimated to be less than $0.3^{\circ}$, we did not correct the measured phase. Figure 14 shows the measured phase shift (shaded dots) together with the calculated values (dashed lines) obtained by the simulation [9] as a function of the bucket number, where the measured phase of the head bunch in the train is a reference. We did not need to take the effect of the phase shift due to the parasitic loss into account, because it should equally act on all bunches. The calculated phase was shifted by $1.6^{\circ}$ for the LER and by $0.2^{\circ}$ for the HER to compare with the measured data. The measured phase and the calculation are quantitatively in good agreement, except at the 

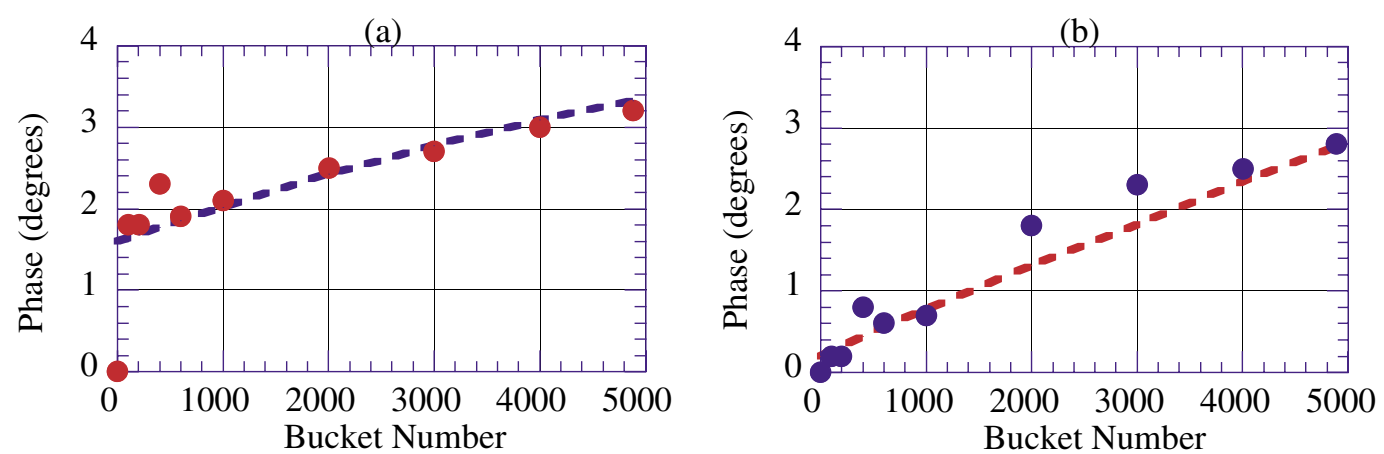

FIG. 14. (Color) Phase shift as a function of the bucket number: (a) LER at $1100 \mathrm{~mA}$ and (b) HER at $800 \mathrm{~mA}$. The phase at the head bunch is the reference. The shaded dots are the measured phase shift and the dashed lines are the calculated phase shift.

leading part of the train. It is seen that the phase in the LER rapidly increases from the head to the bucket number of around 50 (100 ns). After a bucket number of 601, it gradually increases up to the last bunch of the train. The phase difference between the head and the tail bunches is $3.2^{\circ}$ at $1100 \mathrm{~mA}$ in the LER and $2.8^{\circ}$ at $800 \mathrm{~mA}$ in the HER. The phase shift is relatively small for large beam currents, owing to large stored energy in the superconducting cavities [28] and the normal-conducting cavities with energy-storage cavities (ARES) [29]. This small phase shift contributes to a stable operation of $\mathrm{rf}$ systems.

The longitudinal displacement of the collision vertex is obtained from the phase difference at each bucket between the two rings, as given by Eq. (12). The displacement settles within $0.5^{\circ}$ or $0.8 \mathrm{~mm}$. Since the bunch length of $7 \mathrm{~mm}$ is much larger than the displacement, significant luminosity reduction is not to be expected.

The remaining problem is the unexpected rapid increase in the phase observed at the leading part of the train in the LER. The following results have been obtained so far: (i) The phenomenon occurs regardless of a collision effect. (ii) The amount of the phase shift increases as the beam current increases and it also depends on the bunch spacing. (iii) The rise time is about 80 ns (40 buckets). (iv) The bunch oscillation recorder [30] also captures a similar phenomenon. These results suggest that the phase shift may be due to some longitudinal wakes, which are not taken into account in the simulation.

\section{DISCUSSION}

This paper deals with various aspects of beam dynamics: the coupling impedance, the tune shift due to the electron cloud, the beam-beam tune-shift parameter, and the transient beam-loading effect. Discussions are focused on the threshold of the instability due to the electron cloud and the coherent beam-beam parameter with unequal tunes.
According to the two-particle model, the threshold for the blowup in the tune shift is determined by the synchrotron tune. We have examined the threshold in two configurations of solenoids under a constant synchrotron tune of 0.022 . Between the two configurations, 119 solenoids were added in arc sections of the LER [6]. The bunch-by-bunch tune along a train was measured in parallel with the vertical size measurement using an interferometer [31], which obtained an average size for all bunches. In the measurements, the tune rapidly increased at the leading part and tended to saturate at trailing bunches in a train. It was experimentally found that the tune shift depends on the chromaticity, as shown in Fig. 8. Thus, measured data of tunes with different chromaticity should be normalized to the value at zero chromaticity. Figure 15 shows the normalized tune difference between the head bunch and trailing bunches as a function of the LER beam current in the two configurations of solenoids. It can be seen in Fig. 15 that the tune shift measured in configuration 2 (March 2002) slightly reduces at a high current, comparing to that in configuration 1 (December 2001). On the other hand, the measured vertical beam size indicates that the vertical size started to increase at about $1100 \mathrm{~mA}$ in configuration 1 , but, did not increase up to $1200 \mathrm{~mA}$ in configuration 2 [6]. Considering both results of the size measurements and the tune shifts shown in Fig. 15, the tune-shift threshold for the blowup is determined to be about 0.010 . The corresponding cloud density is $1.47 \times 10^{12}\left(\mathrm{~m}^{-3}\right)$. Although the estimated threshold in the tune shift is higher by 0.003 than the calculated threshold of 0.007 using the two-particle model, we can use those tune-shift data as an indicator for the blowup.

The luminosity can be estimated from the vertical beam-beam parameter based on Eq. (8). Figure 16 shows the luminosity obtained from the vertical tune shift together with that obtained from a luminosity monitor. It should be noted that the estimated luminosity uses assumptions that each pair of bunches has an equal luminosity and that the reduction factor is assumed to be $R_{L} / \bar{R}_{y}^{*} \approx 10.0$. Although the estimated luminosity 


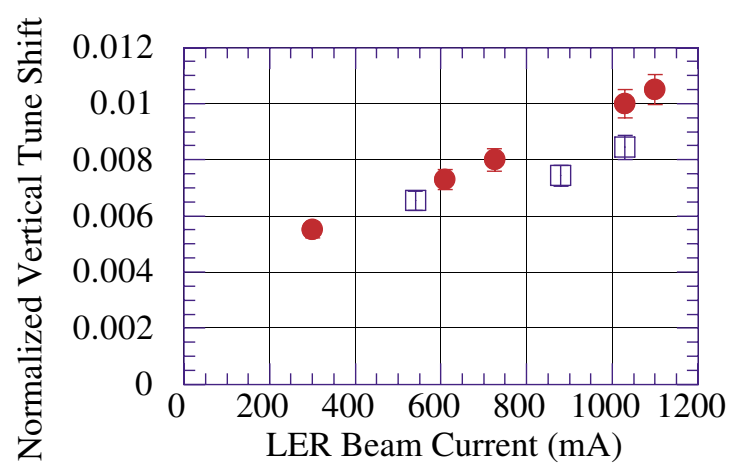

FIG. 15. (Color) Normalized vertical tune shifts in a train as a function of the beam current measured at $\nu_{s}=0.022$. The red dots are tune shifts measured in configuration 1 (December 2001) with chromaticity of 3.3 , and the blue squares are those measured in configuration 2 (March 2002) with chromaticity of 3.7 .

approximately agrees with the measured one, there is a disparity in the current dependence between the luminosity monitor and the tune shift. One candidate for the cause of this disparity is bunch-by-bunch dependence. The measured bunches were the last bunch of a train and were affected by the electron cloud. However, the bunch-by-bunch luminosity monitor [32] did not indicate such dependence. Another candidate is the effect of the nonlinearity in the beam-beam force. The amplitude of excited betatron oscillation should be less than an rms beam size to avoid the nonlinear effects. The vertical tune observed in the HER actually depended on the excitation amplitude and shifted to a lower tune as the excitation amplitude increased. This shift may cause an error in the measurement. Moreover, when a vertical offset

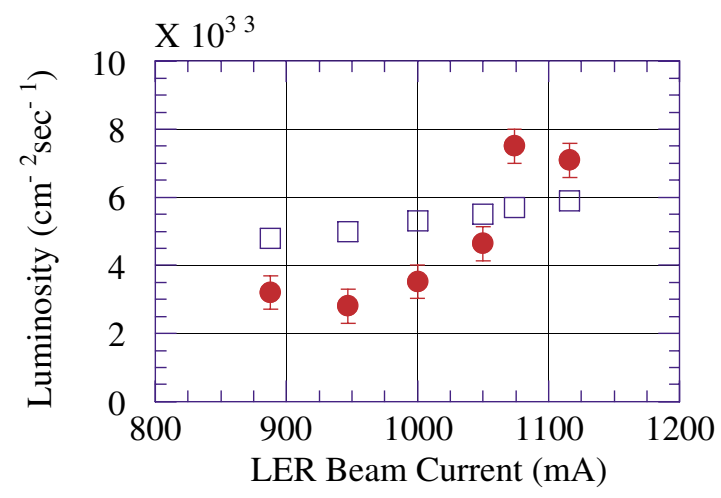

FIG. 16. (Color) The shaded dots indicate the luminosity estimated from the tune shift as a function of the LER beam current, and the squares show that measured by the luminosity monitor. The reduction factors $R_{L} / \bar{R}_{y}^{*}=1.0$ and $\bar{\beta}_{y}^{*}=$ $6.75 \mathrm{~mm}$ were used as parameters for the estimation. The pilot bunch tunes were $\nu_{x 0}^{+}=0.513, \nu_{y 0}^{+}=0.565, \nu_{x 0}^{-}=0.512$, and $\nu_{y 0}^{-}=0.583$. was intentionally made, sidebands and two-horn peaks were observed in the vertical spectra of both rings. These disturbed spectra make it difficult to identify a real tune.

We can estimate the sum of the horizontal emittance from the horizontal beam-beam parameter using Eq. (9). Since the horizontal tunes are almost equal, the Yokoya factor is taken into account in converting the tune shift into the beam-beam parameter, where a Yokoya factor of 1.31 is used. The estimated emittance is $52 \pm 3 \mathrm{~nm} \mathrm{rad}$, assuming $\bar{R}_{x}^{*}=0.7$ [33]. This emittance is larger than the calculated emittance of $42 \mathrm{~nm}$ rad. The disparity may be caused by the dynamic effect, since the horizontal tune is very close to a half integer. According to linear dynamic effects, the perturbed emittance is 1.55 times larger than the original value, when the incoherent beam-beam parameter $\xi_{x}=0.04$. The estimated emittance from the tune shift may be affected by the dynamic effects. It is necessary to confirm the emittance growth using another method.

\section{CONCLUSIONS}

A fast gate that picks up a bunch from a multibunch beam was attached to the turn-by-turn detectors. Precise measurements of individual bunch parameters were performed in a single bunch and in multibunches with and without collision. These measurements were applied to investigate various aspects of the beam dynamics at KEKB.

The measurement in a single bunch gives us information on the coupling impedance. The effective transverse coupling impedance was obtained from the current dependence of the tune shift. The measured value was significantly higher than the design value.

We estimated variations of the electron cloud density along a train from measurements of a tune shift of positron bunches without collision in the LER. The result is consistent with the simulation [15]. The asymmetric contribution due to the solenoids was observed. The reason is not clear so far. The threshold for the blowup in the vertical tune shift indicated a higher value than that for the strong head-tail instability caused by the electron cloud based on the two-particle model.

No significant variation in the tune along a train of electron bunches was observed, but we observed strong current dependence of the tune of \pm 0.03 /A with different signs in the two directions. It is understood to be caused by the fields induced by the resistive-wall current in asymmetric chambers.

The beam-beam tune shift was measured by comparing the tunes of collision and noncollision bunches. Although the luminosity extrapolated from the vertical tune shift roughly agrees with that of the luminosity monitor, the nonlinear effects of the beam-beam force 
and two-horn peaks disturb the tune-shift measurement. The estimated horizontal emittance from the horizontal beam-beam tune shift was larger than the design value. The dynamic effect may contribute to the emittance growth. Further investigation is required.

A precise measurement in the beam phase was performed with a resolution of less than $0.1^{\circ}$. We measured the bunch-by-bunch phase along a bunch train followed by a gap, in order to investigate the transient beam-loading effect. The measured phase shift in a train agrees with the calculation except in the leading part of the train. A rapid advance in the beam phase was observed in the LER, which is considered to be due to the effects of some longitudinal wakes.

\section{ACKNOWLEDGMENTS}

The authors would like to thank Professor K. Oide for his support and fruitful comments. They also thank the members of the KEKB commissioning group for their help.

[1] KEKB Design Report, KEK Report No. 95-7, 1995.

[2] KEK Report No. 2001-157, 2001, edited by E. Kikutani [Nucl. Instrum. Methods (to be published)].

[3] K. Oide, K. Akai, N. Akasaka, K. Bane, A. Enomoto, J. Flanagan, H. Fukuma, Y. Funakoshi, K. Furukawa, J. Haba, S. Hiramatsu, K. Hosoyama, N. Huang, T. Ieiri, N. Iida, T. Kamitani, S. Kato, M. Kikuchi, E. Kikutani, H. Koiso, M. Masuzawa, T. Matsumoto, S. Michizono, T. Mimashi, T. Nakamura, Y. Ogawa, K. Ohmi, Y. Ohnishi, S. Ohsawa, N. Ohuchi, E. Perevedentsev, D. Pestrikov, K. Satoh, M. Suetake, Y. Suetsugu, T. Suwada, M. Tawada, M. Tejima, M. Tobiyama, S. Uno, N. Yamamoto, M. Yoshida, M. Yoshioka, S. Yoshimoto, and F. Zimmermann, in $K E K$ Proceedings 99-24, $e^{+} e^{-}$Factories'99, 2000 (KEK, Tsukuba, 2000), p. 12.

[4] H. Fukuma, K. Akai, N. Akasaka, K. Bane, K. Egawa, A. Enomoto, J. Flanagan, Y. Funakoshi, K. Furukawa, K. Hayashi, S. Hiramatsu, H. Hisamatsu, K. Hosoyama, N. Huang, T. Ieiri, N. Iida, T. Kamitani, K. Kanazawa, S. Kato, M. Kikuchi, E. Kikutani, H. Koiso, S. Kurokawa, T. Mitsuhashi, M. Masuzawa, T. Matsumoto, S. Michizono, T. Mimashi, T. Nakamura, Y. Ogawa, K. Ohmi, Y. Ohnishi, S. Ohsawa, N. Ohuchi, K. Oide, E. Perevedentsev, D. Pestrikov, K. Satoh, M. Suetake, Y. Suetsugu, T. Suwada, F. Takasaki, M. Tanaka, M. Tawada, M. Tejima, M. Tobiyama, T. Tsuboyama, N. Yamamoto, M. Yoshida, M. Yoshioka, S. Yoshimoto, C. H. Yu, and F. Zimmermann, in Proceedings of the 7th EPAC2000, Vienna, 2000 (European Physical Society, Geneva, 2000), p. 1122.

[5] H. Fukuma, K. Akai, N. Akasaka, A. Enomoto, J. Flanagan, Y. Funakoshi, K. Furukawa, S. Hiramatsu, K. Hosoyama, T. Ieiri, N. Iida, T. Kamitani, S. Kato,
M. Kikuchi, E. Kikutani, H. Koiso, T. Kubo, S. Kurokawa, T. Mitsuhashi, M. Masuzawa, T. Matsumoto, T. Mimashi, T. Nakamura, Y. Ogawa, K. Ohmi, Y. Ohnishi, S. Ohsawa, N. Ohuchi, K. Oide, K. Satoh, M. Suetake, Y. Suetsugu, T. Suwada, M. Tawada, M. Tejima, M. Tobiyama, S. Uno, N. Yamamoto, M. Yoshida, M. Yoshioka, S. Yoshimoto, E. Perevedentsev, and F. Zimmermann, in Proceedings of the HEACC2001, Tsukuba, 2001 (KEK, Tsukuba, 2001).

[6] H. Fukuma, J. Flanagan, K. Hosoyama, T. Ieiri, T. Kawamoto, T. Kubo, M. Suetake, S. Uno, S.S. Win, and M. Yoshioka, in Proceedings of the ICFA HB2002 (AIP, New York, 2002).

[7] T. O. Raubenheimer and F. Zimmermann, Phys. Rev. E 52, 5487 (1995).

[8] D. Boussard, in Proceedings of the PAC'91 (IEEE, New York, 1991), p. 2447.

[9] K. Akai, N. Akasaka, K. Ebihara, E. Ezura, M. Suetake, and S. Yoshimoto, in Proceedings of the 6th EPAC, Stockholm, Sweden, 1998 (IOP, Bristol, U.K., 1998), p. 1749.

[10] T. Ieiri and T. Kawamoto, Nucl. Instrum. Methods Phys. Res., Sect. A 440, 330 (2000).

[11] F. Sacherer, in Proceedings of the 9th International Conference on High Energy Accelerators (SLAC, San Francisco, 1974), p. 347.

[12] K. Ohmi, Phys. Rev. Lett. 75, 1526 (1995).

[13] M. Izawa, Y. Sato, and T. Toyomasu, Phys. Rev. Lett. 74, 5044 (1995).

[14] F. Zimmermann, H. Fukuma, and K. Ohmi, CERN Report No. CERN-SL-Note-2000-061 AP, 2001.

[15] F. Zimmermann, CERN Report No. CERN-SL-Note2000-017 AP, 2001.

[16] K. Ohmi and F. Zimmermann, Phys. Rev. Lett. 85, 3821 (2000).

[17] K. Ohmi, S. Heifets, and F. Zimmermann, in Proceedings of the APAC'01, 2001 (IHEP, Beijing, 2001), p. 445.

[18] K. Hirata and E. Keil, Nucl. Instrum. Methods Phys. Res., Sect. A 292, 156 (1990).

[19] K. Yokoya and H. Koiso, Part. Accel. 27, 181 (1990).

[20] M. Tobiyama and E. Kikutani, Phys. Rev. ST Accel. Beams 3, 012801 (2000).

[21] T. Ieiri, K. Akai, and N. Akasaka, in Proceedings of the 12th Symposium on Accelerator Science and Technology, Wako, 1999 (RIKEN, Wako, Japan, 1999), p. 409.

[22] Y. Suetsugu et al., in Proceedings of the EPAC2000, Vienna, 2000 (Ref. [4]), p. 2301.

[23] L. F. Wang, H. Fukuma, K. Ohmi, S. Kurokwa, K. Oide, and F. Zimmermann, ECLOUD02, CERN (to be published).

[24] Y. Shobuda and K. Yokoya (to be published).

[25] R. Nagaoka, in Proceedings of the PAC'01, Chicago, 2001 (IEEE, Piscataway, NJ, 2001), p. 3531.

[26] F.-J. Decker, A. Fisher, L. Hendrickson, K. E. Krauter, B. Murphy, S. Weathersby, and U. Wienands, in Proceedings of the PAC'01, Chicago, 2001 (Ref. [25]), p. 3558.

[27] A. Hofmann, CERN Report No. CERN-SL-99-039 AP, 1999. 
[28] T. Furuya, K. Akai, E. Ezura, K. Hara, K. Hosoyama, A. Kabe, T. Kojima, S. Mitsunobu, Y. Morita, H. Nakai, H. Nakanishi, T. Tajima, and S. Yoshimoto, in Proceedings of the 9th Workshop on $R F$ Superconductivity, 1999 (KEK, Tsukuba, 1999), p. 31.

[29] T. Kageyama, Y. Takeuchi, N. Akasaka, F. Naito, H. Sakai, H. Mizuno, K. Akai, E. Ezura, H. Nakanishi, and Y. Yamazaki, in Proceedings of the 1st APAC, Tsukuba, 1998 (KEK, Tsukuba, 1998), p. 773.
[30] E. Kikutani, J. Flanagan, H. Fukuma, Y. Ohnishi, and M. Tobiyama, in KEK Proceedings 99-24, $e^{+} e^{-}$ Factories'99, 2000 (Ref. [3]), p. 101.

[31] T. Mitsuhashi, J. Flanagan, and S. Hiramatsu, in $K E K$ Proceedings 99-24, $e^{+} e^{-}$Factories'99, 2000 (Ref. [3]), p. 134.

[32] S. Uehara (private communication).

[33] Y. Funakoshi (private communication). 